\title{
GENDER DIFFERENCES WHILE PERFORMING AN EVERYDAY PERCEPTUAL-MOTOR TASK
}

\author{
Muhammad Azam $^{1 *}$, Asif $\mathrm{Ali}^{2}$, Saddam Akbar ${ }^{3}$, Marrium Bashir ${ }^{4}$, Hyun Chae Chung ${ }^{5}$ \\ ${ }^{1 *}$ Assistant Professor, Department of Physical Education and Sports Sciences, Government College University Lahore, \\ Punjab, Pakistan; ${ }^{2}$ Associate Professor, Department of Physical Education and Sports Sciences, Government College \\ University Lahore, Punjab, Pakistan; ${ }^{3,4} \mathrm{Ph}$.D., Scholar, Department of Sports Science, University of Putra Malaysia, \\ Malaysia; ${ }^{5}$ Professor, Department of Sport and Exercise Sciences, Kunsan National University, South Korea. \\ Email: ${ }^{1 *}$ m.azam@gcu.edu.pk, ${ }^{2}$ goraya128@ @otmail.com, ${ }^{3}$ justsaddamakbar4@ gmail.com, ${ }_{5}^{4}$ marriumbshr@gmail.com, \\ ${ }^{5}$ hcx@ kunsan.ac.kr
}

Article History: Received on $18^{\text {th }}$ June 2021, Revised on $27^{\text {th }}$ June 2021, Published on $30^{\text {th }}$ June 2021

\begin{abstract}
Purpose of the study: The aim of this paper was to study gender differences regarding their perceptual judgment and movement behavior in the road crossing task.

Methodology: A simulated road crossing environment outside the Human Motor Behavior laboratory (HMBL) was used to examine the individuals' perceptual-motor behavior. Twenty-four young adults performed the road crossing task in the virtual environment judging whether the available gap was crossable or not crossable and then initiating movement depending on the perceptual information.
\end{abstract}

Main Findings: Participants' gap selection revealed that their cross-ability was influenced by vehicle speed, however, female participants made more errors relative to males. In addition, females took longer to cross and made unnecessary adjustments during crossings. The study findings suggest that females' erroneous perceptual decisions and inconsistent locomotion behavior in road-crossing put them at higher risk relative to their male counterparts.

Application of this study: The findings of this study may apply to developing training programs regarding pedestrian individuals. Training with performing road-crossing tasks may prove to be helpful for refining individuals' perceptual judgment and movement behavior to minimize chances of accidents in road crossing. Specifically, having experience with the road-crossing task in a virtual environment may reduce the tendency towards risk-taking behavior.

The novelty of this study: Most of the past research regarding pedestrian individuals' road crossing behavior examined participants' perceptual judgment (perception) in standing position only or did not analyze movement behavior in the actual walking set up. The approach utilized in our experiment was novel in this regard; individuals can choose to cross a gap and walk wearing a head-mounted display.

Keywords: Pedestrian, Perception-action, Road-crossing, Virtual Environment, Gender Differences.

\section{INTRODUCTION}

Research regarding perceptual-motor skills in the field of Physical Education has a long-lasting historical background. The primary aim of physical education (PE) is developing cognitive, perceptual, and motor skills in the learners from an early age (Zeng et al., 2017). Perceptual-motor skills involve temporal, directional, and spatial aspects of movements throughout the lifespan. In the daily routines of life, we perform several perceptual-motor activities like picking up a cup of coffee, walking through people on the street, catching/hitting a ball in sports, or typewriting. A perceptual-motor skill consists of two components of human behavior, namely, perception and action. Thus, an accurate perceptual-motor performance requires a skilled connection between the perception (the brain/vision) and motor function (the body) of a human while interacting with the environment. To perform a task, a perceiver requires perceiving the relevant information from the environment and initiating movement according to the perceived stimuli.

All sorts of perceptual-motor actions link to the environment that provides numerous opportunities (affordances) to an actor to perform those everyday activities (Gibson, 2014; Stoffregen, 2018). In general, affordances are of two types, namely, stationary and dynamic. Perceiving affordances for stationary objects does not require many adaptations in movement behavior. For instance, a runner in a hurdle race just requires perceiving the place and height of a hurdle on the running track and jump to cross the hurdle accordingly. Namely, the hurdle does not change its place and direction itself because it is a stationary object. On the other hand, perceiving affordances become a more complex task when the objects to be perceived are moving than that of stationary ones because the speed and direction of moving objects change over time (Plumert \& Kearney, 2018). In the context of dynamic affordances, opportunities for an action continuously change over environmental conditions. For example, a gap between two objects could be pass-through-able in a moment but might become unpassable at the next moment due to the change in speed or direction of those objects.

Therefore, the accurate perception-action coupling is fundamental for time-constraint affordances, and it becomes more crucial when performing tasks that may have dangerous consequences. The consequences of misjudging the dynamic affordance or self-abilities can be a collision. An everyday activity of this kind is to perceive and intercept a vehicular gap (i.e., to cross a busy road) that requires carefully making perceptual and movement decisions. 


\section{STUDY OBJECTIVES}

Road crossing is an everyday activity that is potentially dangerous for the pedestrian for all age groups. A body of past research has worked on individuals' road crossing behavior while recording their crossings in real situations or virtual environment settings. However, in the existing research literature, few studies worked on individuals' road-crossing behavior requiring them to perceive while moving. Therefore, the present study aimed at the following objectives.

1. To investigate the individuals' behavior providing them perception and movement opportunity in the virtual road crossing task.

2. To explore gender differences while perceiving and coordinating movement during a road crossing task in the virtual environment.

\section{LITERATURE REVIEW}

\section{Road Crossing (A Perceptual-Motor Activity)}

Road crossing is a routine (perceptual-motor) activity that requires perceiving a crossable gap between moving vehicles and initiating movement according to the traffic environment. For a successful crossing, a road user must effectively utilize perceptual information while choosing a crossable gap (e.g., estimating the speed or distance of approaching vehicles) and to time the self-movement according to the changing traffic environment (Papic, Jovic, Simeunovic, Saulic, \& Lazarevic, 2020). In other words, a road user requires to interrelate the available time with the crossing time and to adjust his/her locomotion for avoiding collision with moving traffic. Thus, this everyday perceptual-motor task (road-crossing) involves perceiving a time gap that affords safe passage through moving vehicles and moving sensibly through the ever-changing traffic environment (Azam, Ali, \& Chung, 2020).

To promote physical culture among youth, children and adolescents are encouraged to adopt "active school transportation (ATS) that denotes walking and biking" (Kontou, McDonald, Brookshire, Pullen-Seufert, \& LaJeunesse, 2020). There are more pedestrian road-users than other modes of travel. (Kontou et al., 2020). A pedestrian, therefore, requires skillfully using perceptual information to choose an inter-vehicle gap and coordinating the self-movement in the duration of crossing action (Dicks, Clashing, O'Reilly, \& Mills, 2016). Making error/s when deciding a crossable gap or coordinating the movement at the wrong time and place may result in a collision with an upcoming vehicle. The alarming consequences of such perceptual errors can be seen in the global report on road safety (WHO, 2018). According to the report, the number of deaths resulting from traffic accidents has reached up to 1.35 million per year. Among other factors to those fatalities, approximately $67 \%$ of road accidents can be attributed to human errors that increasing the number of traffic accidents day by day (WHO, 2018). Such erroneous behavior may occur due to imprecise use of road users' perceptual information and movement timing to cross the road (Azam, Ali, et al., 2020). Minimizing the chances of a collision, therefore, a pedestrian must effectively utilize the perceptual information from the environment (e.g., vehicle speed or its arrival time) and time his/her crossing action according to the changing traffic scenarios.

Human behavior in road crossing tasks may be examined in a real or an artificially devised environment (a prominent field of Ergonomics). Virtual reality (VR) is a technological tool (immersive multimedia) that has been used in several pedestrian simulators (Azam, Choi, \& Chung, 2020; Cavallo, Dommes, Dang, \& Vienne, 2019; Feldstein, Lehsing, Dietrich, \& Bengler, 2018). In virtual reality, a real-like environment can be simulated using computer applications. The use of such an artificially devised environment is not new, even so, its use can be traced back to past research (Lee, Young, \& McLaughlin, 2007). In addition, virtual reality has more benefits than other research apparatuses available in the field for the study of health behavior, education, and training. The most prominent advantage is that it has a little physical risk because individuals can be tested inside a laboratory in an activity for which a real-world situation may be dangerous.

Several research studies investigated how individuals perceive/choose inter-vehicle gaps in real or virtual environments and what factors influence their decisions in a road crossing task. For example, the effects of traffic density on the young and old adults' road crossing decisions and behavior were examined in a virtual environment (Lobjois, Benguigui, \& Cavallo, 2013). In addition, a review study (Feldstein, 2019) has analyzed several factors that may affect perceptual judgment in road crossing tasks such as human factors (age, gender, etc.), traffic-environment factors (observation time, spatial distance, and vehicle velocity, etc.), and technical factors (screen display contrast, stereoscopy, and field of view, etc.,). Moreover, the results of a recent study involving walk on a treadmill revealed age differences between children and young adults regarding perceptual-motor behavior in a road crossing task (Chung, Choi, \& Azam, 2020). Partial results of a pedestrian simulator validation study revealed that there were no significant gender differences when young adults were examined in a road crossing learning task (Azam, Ali, et al., 2020). Exempting the learning task, therefore, we were further keen to know whether gender differences regarding perception-movement behavior emerge when individuals perceive/walk to cross moving gaps in a virtual traffic environment. Working on this intention, we reviewed the previous studies on the relevant task focusing on gender as a factor. 


\section{Gender Differences in Road Crossing}

The evidence already established that several factors affect gap acceptance and road crossing behavior. Among them, gender is an important human factor and the effects of gender have been investigated by many human factor psychologists or transportation researchers so far. Earlier evidence suggested that behavioral differences regarding gender in road-crossing emerged because females make more perceptual errors than males when deciding crossable gaps (Manser \& Hancock, 1996).

Past work involving estimation tasks on pedestrian road crossing decisions reported that males' and females' behavior was different when answering "Yes" for a crossable gap and "No" for a not crossable gap between moving vehicles (Holland \& Hill, 2010). A couple of studies (Lobjois \& Cavallo, 2007, 2009) worked on age differences in road crossing decisions and included gender as a between-participant factor. The partial results of those studies revealed significant gender differences regarding gap estimation among three age groups (i.e., children, young adults, and elderly). Male participants' reaction time and crossing time were faster than females, and they (males) choose time gaps sensibly than females. Also, the male and female perceivers behaved dissimilarly when they were observed directly in the real crossing situation (Tom \& Granie, 2011). Similarly, a study based on filmed records of pedestrian crossings demonstrated that females made more risky decisions and left with less time to spare than males (Holland \& Hill, 2010$)$. Partial results of another study on crossing decisions revealed no significant gender differences when the participants were required to choose time-to-contact (TTC) of the upcoming vehicle in a real vs. virtual environment (Feldstein \& Dyszak, 2020). An important thing, in this respect, is the type of experimentation or a paradigm that should also be considered while examining individuals' behavior.

Notably, most of the previous studies examined individuals' road-crossing behavior requiring them to judge the estimated time of vehicle arrival at the crossing position (Feldstein \& Dyszak, 2020; Manser \& Hancock, 1996). Besides, studies involved participants in passive perception requiring them to answers (Yes/No) while standing (Lobjois \& Cavallo, 2007) or used filmed records (Holland \& Hill, 2010). In contrast, action-relevant movement facilitates an actor with the opportunity to identify the perceptual information from the environment optimized by locomotion (Lobjois et al., 2013). This perception-movement assumption could be traced back to a study that investigated the role of perception-movement on perceivers' catchability (Oudejans, Michaels, Bakker, \& Dolne, 1996) to catch a flying ball at the right time and place. The same assumption is applicable when intercepting a moving vehicular gap.

\section{The Ecological Approach of Perception-Action}

The ecological approach of perception-action was introduced by James J. Gibson in 1979. Gibson's ecological approach annotates that perception is coupled with movement (e.g., movement of receptors, limbs, and the entire animal). Namely, perception and action evolve a continuous cycle in which "we perceive in order to move, but we also move in order to perceive" (Gibson, 2014). The ecological approach demonstrates how perceptual-motor control counteracts during interactions between animals/humans (i.e., pedestrians in our study) and inanimate physical systems (i.e., the road involved in the task). Moreover, behavioral dynamics "in a consistent approach have been proposed to account for the dynamics of perception and action" (Warren, 2006). Following Gibson's idea, "the behavioral dynamics approach annotated that rather than being localized in an internal (or external) structure, control is distributed over the actorenvironment system". That is why the inclusion of an "actor-environment system" is fundamental in the circumstance of the present pedestrian road crossing task.

Pedestrians' behavioral responses in street crossing tasks can be explained in more detail under the assumption of affordance-based control (Fajen, 2007). Concerning visually guided actions, the model stated that; "the primary function of perception is to make it possible for the actors to see the environment in terms of 'what they can and cannot do' and that the successful performance depends on the perception of possibilities for action” (p. 384). It is, therefore, necessary that pedestrians must be able to distinguish between a crossable and/or not crossable gap based on the actor environment system.

In addition, the ecological approach of perception-action signified the role of the performers' movement to pick up the perceptual information from the environment (Oudejans et al., 1996). The energy arrays are co-structured during a performer's movement, when a performer moves his/her orientation or displacement in the environment is specified based on those structured patterns of energy. Perception in the standing-still position does not provide a participant with the opportunity to refresh information from the environment. Nonetheless, the representativeness of the "actorenvironment system" in experimental paradigms is crucial. The experimental paradigms developed based on this assumption (perception-action coupling) allow participants to receive and utilize the information available through the environment to support their motor responses (Warren, 2006).

Under this ecological tenet, movement is crucial, and moving actively in such tasks generates more calibration in perception and action system than that of the standing position. Perception-movement assumption was not fully utilized in the previous studies reviewed above on our topic of interest. The current technological advancements brought several changes in the previously used simulations that is why the VRs being used in the present era are more immersive, interactive (Azam, Choi, et al., 2020; Feldstein \& Dyszak, 2020). Utilizing such innovative and interactive VR, the 
present study focused on examining individuals' gender differences in their perceptual judgment and walking behavior when they walk to cross the road through the simulated environment.

\section{HYPOTHESES OF THE STUDY}

The perception-movement approach was employed in our study to assess individuals' perceptual judgment and walking behavior in a virtual environment. Based on the findings of previous studies, it was expected that females may make more errors due to differences in cognitive abilities or move fast striving for greater safety margins than males (Montgomery, Kusano, \& Gabler, 2014). The hypotheses of our study were as follows.

$\mathbf{H}_{0}$ : There are no significant differences between young male and female individuals regarding perceptual-motor behavior in the road crossing task.

$\mathbf{H}_{1}$ : Females make more errors while choosing gaps and coordinating movement than males in the road crossing task.

$\mathbf{H}_{2}$ : Females make less accurate adjustments in walking speed than males when they walk to cross a road in the virtual environment.

\section{METHODOLOGY}

This experimental study involved undergraduate students from the Kunsan National University (age range: 20 28). We did not use any athlete as a participant because the task requires no athletic experience. The experiment was conducted outside the human motor behavior laboratory (HMBL) situated in the Department of Sports and Exercise Sciences of Kunsan National University (KNU). The sample of normal students consisted of 12 males ( $\mathrm{M}=22.80, \mathrm{SD}=2.57 \mathrm{yrs})$ and 12 females $(\mathrm{M}=23, \mathrm{SD}=2.08 \mathrm{yrs})$ with normal/corrected to normal vision. The participants were randomly selected from the different departments of the KNU. Ethical approval to experiment was attained from the university's Research Ethics Committee (REC). Before participating in the experiment, we provided all the participants with written informed consent forms according to the Declaration of Helsinki (1964).

\section{Apparatus and task}

To examine the perceptual-motor behavior of individuals in the road crossing task, the study used the pedestrian simulator (Figure $1 \& 2$ ) developed by an interdisciplinary research group (Azam, Choi, et al., 2020). The parts of the simulator were a real experimental street (10m long and $5 \mathrm{~m}$ wide), an Intel computer (3.30 GHz, 8.00 GB RAM), an Oculus Rift (a head-mounted display: HMD) with a RUNA smartphone, and Arduino Uno.

The RUNA smartphone inserted into HMD was linked to the computer $\rightleftarrows$ Arduino Uno via Wi-Fi and Bluetooth. The Arduino Uno was equipped with a $30 \mathrm{~m}$ long spring-loaded wire to measure the forward locomotion of a pedestrian. The computer system was the source of data storage and served as a control station.

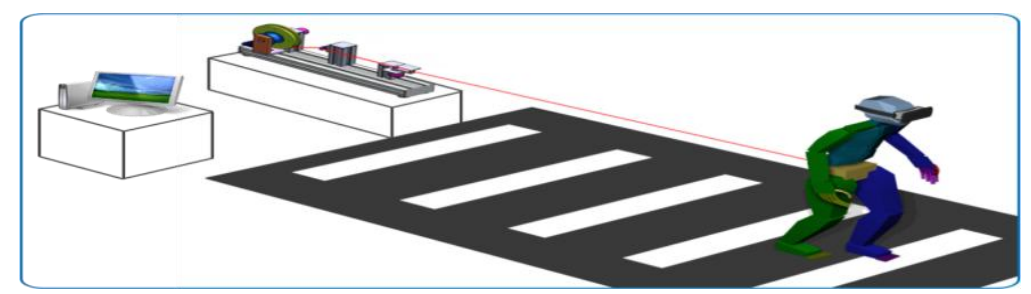

Figure 1: A cartoon image of the pedestrian simulator. (reused with permission from (Azam, Choi, et al., 2020))

The virtual environment consisted of a two-lane road divided by yellow lines. The total width of the virtual road was $7 \mathrm{~m}$ (3.5 m width of each lane). The sky, roadside trees, and residential buildings were depicted in the virtual scene representing a nature-like view. Two (sedan-sized) cars were visible on the virtual road from the left side. The virtual view provided participants with three-dimensional (3-D) stereoscopic visual imagery.



Figure 2: Image of the virtual scene. left: pre-starting of a trial, right: post-starting of a trial scene. (reused with permission from (Azam, Choi, et al., 2020)) 
A participant's task was to judge whether the gap crossable or not. The participants were instructed, "after making a decision, you require walking to cross the gap if crossable and do not walk if the gap is not crossable". They were further instructed to walk unless a success or crash sign appears on the display screen of the HMD.

\section{Procedure and Design}

The participants, upon arrival in the laboratory, were briefed about the task. Following receiving instructions, each of the participants participated in a familiarization session. The purpose of the familiarization session was to make the participant familiar with the environment and the task. The familiarization session consisted of a free walk trial without wearing the HMD, three trials with no traffic wearing the HMD, and two trials with cars at two different speeds (i.e., 25 $\mathrm{km} / \mathrm{h}, 70 \mathrm{~km} / \mathrm{h}$ speed).

Before starting a trial, the participant stands wearing the HMD and facing towards the virtual road viewable through the HMD. A participant could start walking at the "Go" command given by the experimenter. On the "Go" command, the participant must immediately look left to see upcoming cars. The signs of success, crash, or failure are displayed on the screen as outcome feedback when a trial ends.

Six-gap times ranging from $2 \mathrm{~s}$ to $4.5 \mathrm{~s}$ (0.5-s increments) were randomized between the two cars. Among those time gaps, we included both crossable and not crossable conditions to examine individuals' ability to distinguish between them. Those time gaps were presented to participants using two speeds, the cars' speed was either $36 \mathrm{~km} / \mathrm{h}$ or $55 \mathrm{~km} / \mathrm{h}$, respectively. This combination ( 6 gaps $\times 2$ speeds) comprised of 12 experimental conditions in total.

\section{Data Analysis}

The simulation recorded pedestrians' outcomes of crossing decisions. The possible outcome of a trial could be classified as a success, collision, or failure. Success: when a pedestrian crossed the gap without colliding any vehicle. Collision: when a pedestrian collided with any of the two cars (either with lead or rear car). Failure: when a participant missed or did not accept some gap. Other coordinates were a pedestrians' crossing time from the start of a trial to the interception point, the time of both the lead and rear car from starting to arriving at the crosswalk, and pedestrians' walking velocity $(\mathrm{m} / \mathrm{s})$. First, we refined the raw data in the MATLAB software and then included the following variables in the statistical analysis based on the refined data.

Firstly, participants' gap crossing thresholds from not-cross to cross were calculated for males and females separately depending on the outcome data. A binary logistic regression analysis was used for this purpose, all collisions and failures were classified as not to cross (No), and all successful crossings were to cross (Yes). The binary measures indicated between 0 and 1 for the outcome data. The logistic function that was utilized for calculating critical points and thresholds is as follows; $\mathrm{F}(\mathrm{x})=1 /\left(1+e^{\wedge}(-((x-\alpha) / \beta))\right)$ where $\mathrm{x}=$ time gap and $\beta=$ slope of the logistic curve at point $\alpha$. The equation determined the critical values and thresholds. The critical values indicate the points where pedestrians' behavior changes from not cross to cross, i.e., where the probability of crossings was $0.5(50 \%)$. The thresholds for crossing indicate the abruptness of the transition that corresponds to the distance between $25 \%$ and $75 \%$ of the responses.

Secondly, we calculated the crossing time of the individuals. Crossing time: the total time duration beginning from when a participant was asked to go and the time when he/she reached the end-of-first lane. The mean crossing was computed for both genders based on the successful crossings.

Thirdly, we calculated the individuals' crossing decisions, because a pedestrian requires accurately perceiving the gap that affords safe crossing and avoiding a collision in road crossing situations. Therefore, we compared the misjudged crossing decisions between males and females to find the difference in this regard. For this purpose, we divided the misjudged crossing decisions into two commonly known categories, i.e., collisions and failures as categorized in a previous study (Lobjois \& Cavallo, 2009).

Lastly, since we utilized the perception-action approach, we also included the walking velocity of the participants in our analysis to compare how males and females move/walk differently during crossing action. For this purpose, we segmented participants' walking speed into $1 \mathrm{~s}$ intervals starting from the initiation to the end of the trials. Removing the first $0.5 \mathrm{~s}$ interval, it becomes five intervals for each trial.

The statistical analyses used were a binary logistic regression for critical points and thresholds, an analysis of variance (ANOVA) for crossing time and walking velocity, and a chi-square analysis for calculating the percentage of misjudged crossings, respectively. The researchers used a 0.05 significance level for all statistical analyses mentioned above.

\section{RESULTS}

\section{Crossing Thresholds}

Logistic regression was performed to evaluate the participants' gap selection behavior. The analysis was completed using 2 car speeds $\times 2$ genders. For $36 \mathrm{~km} / \mathrm{h}$ car speed, the logistic model was significant for females: $\chi^{2}(1)=34.625, p<$ .0005 , explaining $R^{2}=54.4 \%$ of variance in the gap selection. The logistic model was also significant for males: $\chi^{2}(1)=$ 
$16.707, p<.0005$, explaining $R^{2}=32.7 \%$ of variance in the gap selection. The critical points were $3.25 \mathrm{~s}$ for females and $3.56 \mathrm{~s}$ for males, respectively. It shows that females were accepting shorter gaps than men at slower car speeds.

For $55 \mathrm{~km} / \mathrm{h}$ speed, the model was significant for females: $\chi^{2}(1)=35.447, p<.0005$, explaining $R^{2}=55.7 \%$ of variance in the gap selection. The model was also significant for males: $\chi^{2}(1)=32.482, p<.0005$, explaining $R^{2}=55.8 \%$ of variance in the gap selection. The critical points and thresholds are shown in Table1.

Table 1: Showing the participants' critical points (in seconds) where the transition alters from not cross to cross decisions.

\begin{tabular}{llll}
\hline Car Speed & Gender & Critical Points & Threshold \\
\hline $36 \mathrm{~km} / \mathrm{h}$ & Female & $3.25 \mathrm{~s}$ & $0.46 \mathrm{~s}$ \\
\hline & Male & $3.56 \mathrm{~s}$ & $0.41 \mathrm{~s}$ \\
\hline $55 \mathrm{~km} / \mathrm{h}$ & Female & $3.44 \mathrm{~s}$ & $0.40 \mathrm{~s}$ \\
\hline & Male & $3.30 \mathrm{~s}$ & $0.45 \mathrm{~s}$ \\
\hline
\end{tabular}

\section{Crossing Time}

Crossing time from all accepted crossings was analyzed using a 2 (gender: male, female) x 2 (car speed: $36 \mathrm{~km} / \mathrm{h}, 55$ $\mathrm{km} / \mathrm{h}$ ) repeated measures analysis of variance (ANOVA). The analysis showed a main effect of gender: $\mathrm{F}(1,103)=$ 4.010, $p<.048, \eta^{2} \mathrm{p}=.037$. Mean crossing time for females was significantly greater $(4.41 \pm 0.27 \mathrm{~s})$ than males $(4.31 \pm$ $0.30 \mathrm{~s})$ that shown in Figure 3. We also found a main effect of car speed $\mathrm{F}(1,103)=4.166, p<.044, \eta_{\mathrm{p}}^{2}=.039$. Mean crossing time of participants was shorter $(4.31 \pm 0.27 \mathrm{~s})$ when cars traveled at the speed $36 \mathrm{~km} / \mathrm{h}$ compared to $(4.41 \pm$ $0.30 \mathrm{~s}$ ) when speed was $55 \mathrm{~km} / \mathrm{h}$.

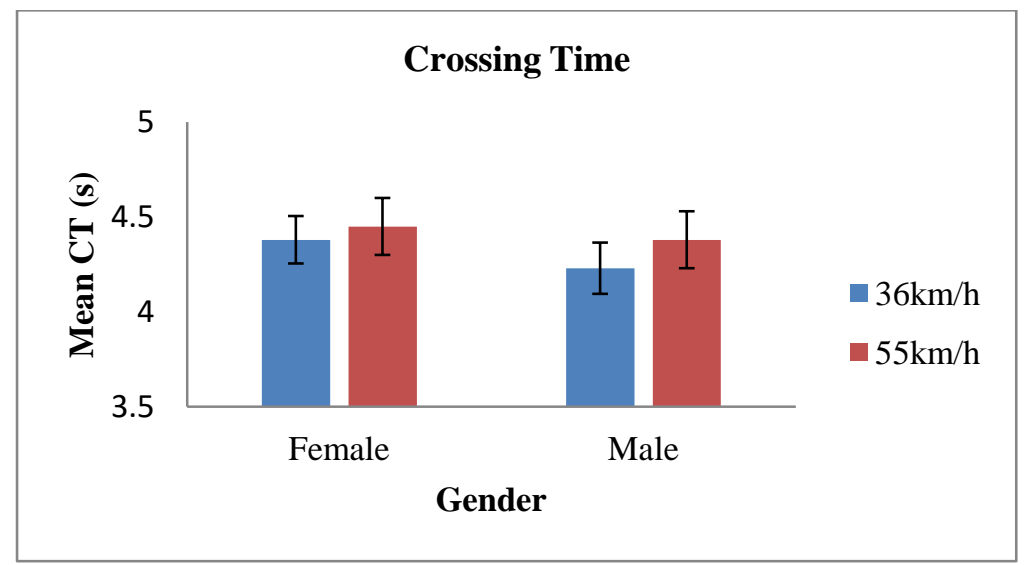

Figure 3: Mean crossing time (in seconds) as a function of gender for both car speeds. (CT = Crossing Time)

\section{Crossing Decisions}

We compared individuals' crossing decisions to evaluate the gender differences at both car speeds. We analyzed crossing decisions using a 2 (gender: males, females) x 2 (car speed: $36 \mathrm{~km} / \mathrm{h}, 55 \mathrm{~km} / \mathrm{h}$ ) chi-square analysis on the number of erroneous crossing decisions and failure to cross.

The analysis showed that the number of errors was marginally significant for gender $\left(\chi^{2}(2)=5.639, p=.060\right)$. The number of female collisions was larger (40) than that of males (25). The number of failures of females was less (7) than that of males (12). The number of misjudged crossings for car speed showed in figure 4 .

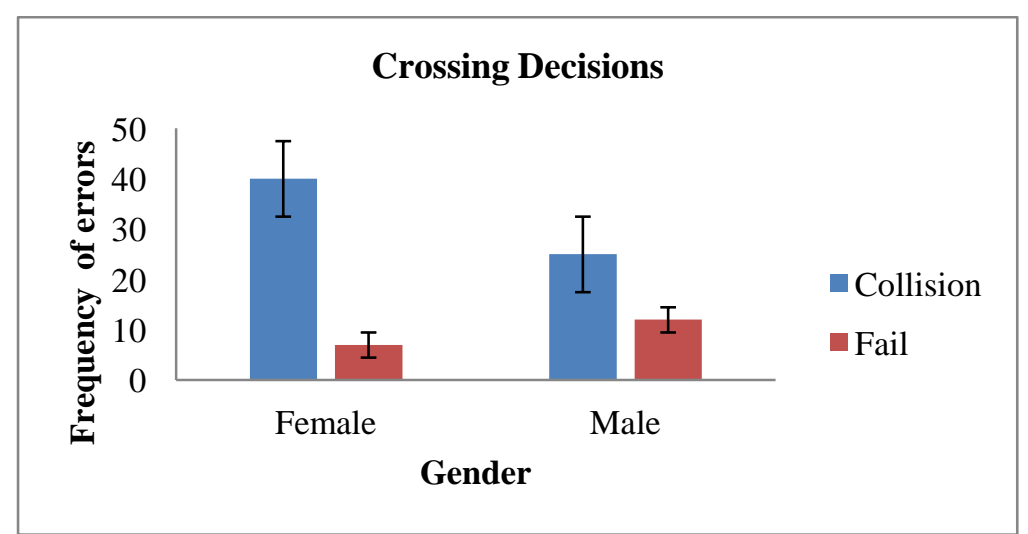

Figure 4: The number of misjudged crossing decisions as a function of gender. 


\section{Walking Velocity}

In the next step, we compared participant's walking velocity regarding adjustments in their locomotion throughout crossing action. The segmented velocity data were entered into repeated measures ANOVA using a 2 genders x 2 car speeds design. Degrees of freedom were corrected using Greenhouse-Geisser. There was a significant interaction between walking velocity and gender: Walk's Lambda $=0.947, \mathrm{~F}(4,227)=3.177, p<.014, \eta^{2} \mathrm{p}=.053$. The interaction between walking velocity at car speed was also significant, $\mathrm{F}(4,227)=3.426, p<.048, \eta^{2} \mathrm{p}=.015$. Following are the walking velocity patterns of both genders.
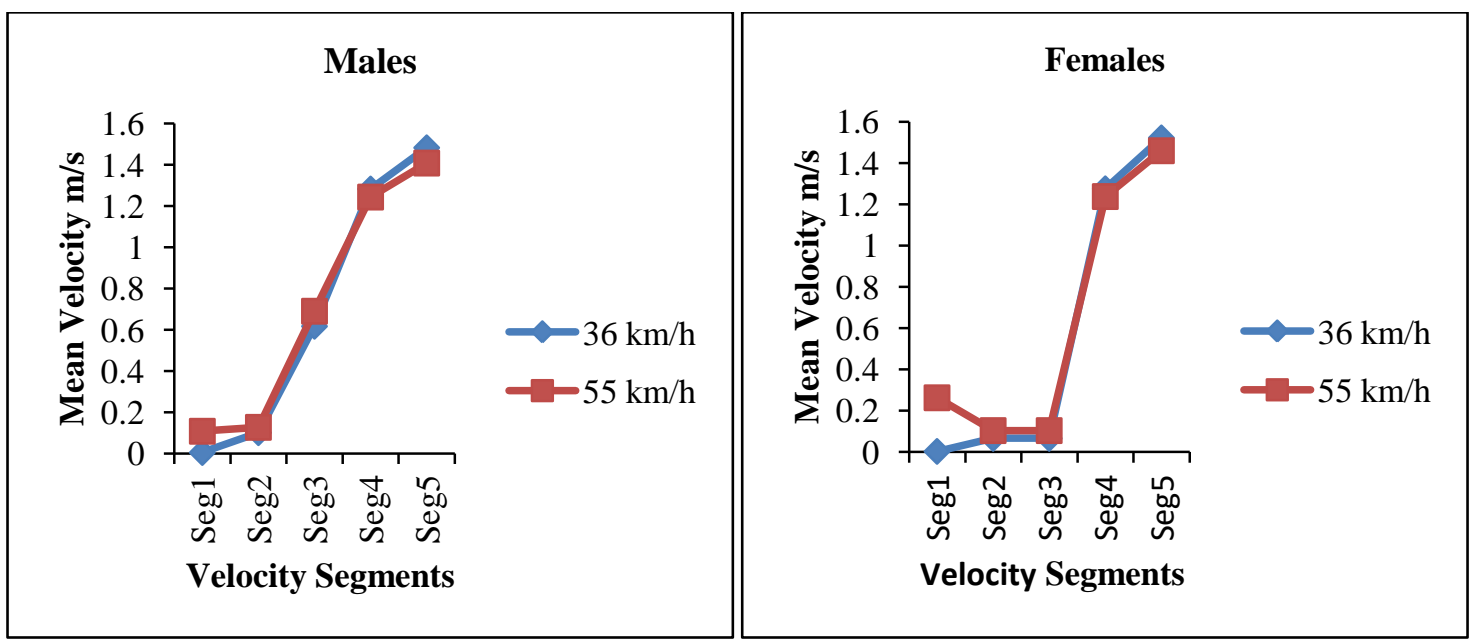

Figure 5: Mean walking velocity as a function of segmented data for gender at both car speeds (left males, right females)

\section{DISCUSSION}

The present study examined pedestrian's perceptual judgment and walking behavior in a road-crossing task using an interactive virtual environment that involves participants' perception-action system instead of perceptual judgments alone. Secondly, the study examined gender differences in road-crossing behavior. The analysis regarding gender differences in gap thresholds clearly showed that individuals' gap selection was different at both vehicle speeds. Females selected shorter gaps than males when cars traveled at a slower speed $(36 \mathrm{~km} / \mathrm{h})$. On the other hand, females were inclined to accept larger gaps relative to males at a higher car speed $(55 \mathrm{~km} / \mathrm{h})$. Overall, the results involving gap selection indicated that the perception of both genders influenced more as the higher car speed.

In specific, gender differences in gap selection emerged from the results of critical points and thresholds indicating a transition from not cross to cross at both vehicle speeds that were not clearly explained in previous research (Lobjois \& Cavallo, 2007, 2009).

The second aspect, of our analysis, was the participants' percentage of gap selection at both vehicle speeds (slower $36 \mathrm{~km} / \mathrm{h}$, faster $55 \mathrm{~km} / \mathrm{h}$ ). Females' percentage of gap selection was higher than males at slower vehicle speed, However, that behavior in gap selection showed contradiction when vehicles traveled at a higher speed. The percentage of females' gap selection decreased than males at high vehicle speed. Importantly, change (decrement) in females' behavior regarding gap selection at higher vehicle speed was an interesting aspect of our results contrary to the prior findings (Lobjois \& Cavallo, 2007). Such behavior indicated that females' gap selection was affected by vehicle speed more than their male counterparts because they accepted shorter gaps even at higher vehicle speed. Based on this indication, it could be assumed that females were not considering the speed of the vehicles, but they seemingly relied on inter-vehicle distance (Morrongiello, Corbett, Milanovic, Pyne, \& Vierich, 2015), however, further research is necessary to explain this aspect of road-crossing behavior while working on gender differences.

Further, our results also demonstrated gender differences relating to gap crossing time. Females took a longer time to cross than males showing differences in individuals' movement capabilities. In addition, the results of misjudgment behavior also revealed gender differences in decision-making behavior. In addition, the results of collisions showed that females took more risky decisions than males when they cross the road in a virtual environment that support the previous findings (Lobjois et al., 2013; Lobjois \& Cavallo, 2007, 2009). The percentage of misjudged crossings indicated that females made more unsafe crossings relative to males. Based on previous work regarding gender differences in perception of vehicle arrival time and road-crossing decisions (Holland \& Hill, 2010), the present research also demonstrated that females behaved differently than males in this everyday perceptual-motor task revealing more misjudged crossing decisions.

The behavior regarding walking velocity also showed that females' approaching velocity was not consistent with the constant bearing angle, but it showed abrupt adjustments in their locomotion. From female behavior, it appears that they 
were not adjusting their walking speed according to the environmental stimuli. On the other hand, males walked smoothly increasing their velocity throughout the gap interception. This behavior shows that they are trying to keep consistent with the constant bearing angle strategy.

An important thing that is to be considered in our results was a larger number of collisions. In this respect, one possibility could be participants' tendency towards taking risky decisions in a safe (game-like) virtual environment. The second possible reason, in this regard, might be the effect of the so-called 'Hikikomori Culture'. It is "a form of severe social withdrawal that has been frequently described in Japan and is characterized by adolescents and young adults who become recluses in their parents' homes" ((Teo \& Gaw, 2010), p. 444). That could affect the perceptual-motor behavior of participants because the sample involved young adults from Asiatic societies. Another possible explanation of this erroneous behavior is possible that the individuals could not utilize perception and action calibration without having experience about the consequences of crossing action in the virtual environment. However, practical experience or short training (Azam, Ali, et al., 2020) might be considered for calibrating perceptual judgment and movement behavior to minimize the incidence of errors in the road-crossing task.

Furthermore, several other factors might be considered in more detail how those gender differences emerged in perceptual judgment and movement behavior. Males who have more driving experience on road than females (Kontou et al., 2020) may benefit from a more accurate perception of gap affordance. Else, gender differences in such coordinative tasks may emerge based on social issues/experiences (Meyers-Levy \& Loken, 2015). In addition, gender differences emerged into interceptive skills related to hitting/catching a ball suggests various patterns of visual adaptation between both genders (Moreno-Briseno, Diaz, Campos-Romo, \& Fernandez-Ruiz, 2010). In short, it is pertinent that gender differences emerged in this perceptual-motor task (i.e., road crossing) due to individuals' developmental perspective and experience that they derived from performing such everyday interceptive skills in the daily routine of life.

The implications of the findings of this study may apply to develop training programs for pedestrian individuals. Because, children and adolescents (Plumert, Kearney, Cremer, Recker, \& Strutt, 2011) are not much skilled in differentiating between crossable or not crossable gaps. Likewise, the elderly suffer a decline in perceptual-motor capabilities with age (Lobjois et al., 2013). Therefore, experience with performing the road-crossing task may prove to be helpful for refining perceptual judgment and the movement behavior during crossing action. In specific, experience with the task can reduce the tendency towards risk-taking behavior under the knowledge of the result of a crossing action. Also, practice can increase control over the movement timing through interrelating the pedestrians' gap choices and movement coordination while having experience with the crossing task. Timing of movement is crucial and more control on movement timing is necessary for the avoidance of pedestrian-vehicle collisions.

\section{CONCLUSION}

In the concluding remarks, it is pertinent to consider the paradigms with maximum ecological validity that may provide more natural responses of participants involving their perception-action system functionally. As expected in our study, misjudged crossing decisions and walking behavior showed that girls made more unsafe road crossing decisions than boys did. This kind of behavior suggested that female pedestrians perceive-act differently than their male counterparts in the road crossing task that put them at more risk. In addition, the walking simulator utilized in the present study is a promising instrument for the studies involving human perceptual-motor behavior in road crossing.

\section{LIMITATION AND FUTURE DIRECTIONS}

Our study was limited to involve young adults for testing gap affordance and movement behavior in the road-crossing task. However, a road crossing situation is crucial for all age groups and is not confined to a specific age group. Further work is needed to understand pedestrians' behavior by enlarging the sample size or extending the investigations involving all developmental stages (children, adolescents, and young adults). In addition, movement initiation, movement strategy, and crossing time on this dynamic gap affordance task may be considered in future research studies.

\section{ACKNOWLEDGEMENT}

Authors acknowledge the funding and apparatus provided by the Korea Institute for Advancement of Technology, and the Ministry of Trade, Industry, and Energy [grant number: 10044775].

\section{AUTHORS CONTRIBUTION}

The first author (Muhammad Azam) developed the idea and prepared the first draft of the study. The second author (Asif Ali) helped in improving the discussion section. The third and fourth authors (Saddam Akbar and Marrium Bashir) assisted in revising the whole manuscript. The fifth author (Hyun Chae Chung) was the study advisor.

\section{REFERENCES}

1. Azam, M., Ali, A., \& Chung, H. C. (2020). Whether short-term experience can enhance perceptual-motor learning in a moving (vehicular) gap interception task. The Spark, 5(1), 13-27. 
2. Azam, M., Choi, G., \& Chung, H. C. (2020). An HMD-based pedestrian simulator for training and measuring individuals perceptual-motor behaviour in road crossing. International Journal of Human Factors and Ergonomics, 7(4), 325-342. https://doi.org/10.1504/IJHFE.2020.112501

3. Cavallo, V., Dommes, A., Dang, N.-T., \& Vienne, F. (2019). A street-crossing simulator for studying and training pedestrians. Transportation Research Part F: Traffic Psychology and Behaviour, 61, 217-228. https://doi.org/10.1016/j.trf.2017.04.012

4. Chung, H. C., Choi, G., \& Azam, M. (2020). Effects of initial starting distance and gap characteristics on children's and young adults' velocity regulation when intercepting moving gaps. Human Factors, 62(6), 10021018. https://doi.org/10.1177/0018720819867501

5. Dicks, M., Clashing, C., O'Reilly, L., \& Mills, C. (2016). Perceptual-motor behaviour during a simulated pedestrian crossing. Gait \& Posture, 49, 241-245. https://doi.org/10.1016/j.gaitpost.2016.07.003

6. Fajen, B. R. (2007). Affordance-based control of visually guided action. Ecological Psychology, 19(4), 383410. https://doi.org/10.1080/10407410701557877

7. Feldstein, I. T. (2019). Impending collision judgment from an egocentric perspective in real and virtual environments: a review. Perception, 48(9), 769-795. https://doi.org/10.1177/0301006619861892

8. Feldstein, I. T., \& Dyszak, G. N. (2020). Road crossing decisions in real and virtual environments: A comparative study on simulator validity. Accident Analysis \& Prevention, 137, 105356. https://doi.org/10.1016/j.aap.2019.105356

9. Feldstein, I. T., Lehsing, C., Dietrich, A., \& Bengler, K. (2018). Pedestrian simulators for traffic research: state of the art and future of a motion lab. International Journal of Human Factors Modelling and Simulation, 6(4), 250-265. https://doi.org/10.1504/IJHFMS.2018.096128

10. Gibson, J. J. (2014). The ecological approach to visual perception: classic edition: Psychology Press. https://doi.org/10.4324/9781315740218

11. Holland, C., \& Hill, R. (2010). Gender differences in factors predicting unsafe crossing decisions in adult pedestrians across the lifespan: a simulation study. Accident Analysis \& Prevention, 42(4), 1097-1106. https://doi.org/10.1016/j.aap.2009.12.023

12. Kontou, E., McDonald, N. C., Brookshire, K., Pullen-Seufert, N. C., \& LaJeunesse, S. (2020). U.S. active school travel in 2017: Prevalence and correlates. Preventive Medicine Reports, 17, 101024. https://doi.org/10.1016/i.pmedr.2019.101024

13. Lee, D. N., Young, D. S., \& McLaughlin, C. M. (2007). A roadside simulation of road crossing for children. Ergonomics, 27(12), 1271-1281. https://doi.org/10.1080/00140138408963608

14. Lobjois, R., Benguigui, N., \& Cavallo, V. (2013). The effects of age and traffic density on street-crossing behavior. Accident Analysis \& Prevention, 53, 166-175. https://doi.org/10.1016/j.aap.2012.12.028

15. Lobjois, R., \& Cavallo, V. (2007). Age-related differences in street-crossing decisions: the effects of vehicle speed and time constraints on gap selection in an estimation task. Accident Analysis \& Prevention, 39(5), 934943. https://doi.org/10.1016/j.aap.2006.12.013

16. Lobjois, R., \& Cavallo, V. (2009). The effects of aging on street-crossing behavior: from estimation to actual crossing. Accident Analysis \& Prevention, 41(2), 259-267. https://doi.org/10.1016/j.aap.2008.12.001

17. Manser, M. P., \& Hancock, P. A. (1996). Influence of approach angle on estimates of time-to-contact. Ecological Psychology, 8(1), 71-99. https://doi.org/10.1207/s15326969eco0801_4

18. Meyers-Levy, J., \& Loken, B. (2015). Revisiting gender differences: What we know and what lies ahead. Journal of Consumer Psychology, 25(1), 129-149. https://doi.org/10.1016/j.jcps.2014.06.003

19. Montgomery, J., Kusano, K. D., \& Gabler, H. C. (2014). Age and gender differences in time to collision at braking from the 100-Car Naturalistic Driving Study. Traffic injury prevention, 15 Suppl 1(sup1), S15-20. https://doi.org/10.1080/15389588.2014.928703

20. Moreno-Briseno, P., Diaz, R., Campos-Romo, A., \& Fernandez-Ruiz, J. (2010). Sex-related differences in motor learning and performance. Behavioral and Brain Functions, 6(1), 74. https://doi.org/10.1186/1744-9081$\underline{6-74}$

21. Morrongiello, B. A., Corbett, M., Milanovic, M., Pyne, S., \& Vierich, R. (2015). Innovations in using virtual reality to study how children cross streets in traffic: evidence for evasive action skills. Injury Prevention, 21(4), 266-270. https://doi.org/10.1136/injuryprev-2014-041357

22. Oudejans, R. R., Michaels, C. F., Bakker, F. C., \& Dolne, M. A. (1996). The relevance of action in perceiving affordances: perception of catchableness of fly balls. Journal of Experimental Psychology: Human Perception Performance, 22(4), 879-891. https://doi.org/10.1037//0096-1523.22.4.879

23. Papic, Z., Jovic, A., Simeunovic, M., Saulic, N., \& Lazarevic, M. (2020). Underestimation tendencies of vehicle speed by pedestrians when crossing unmarked roadway. Accident Analysis \& Prevention, 143, 105586. https://doi.org/10.1016/j.aap.2020.105586

24. Plumert, J. M., \& Kearney, J. K. (2018). Timing Is Almost Everything: How Children Perceive and Act on Dynamic Affordances. Advances in Child Development and Behavior, 55, 173-204. https://doi.org/10.101 6/bs.acdb.2018.05.002

25. Plumert, J. M., Kearney, J. K., Cremer, J. F., Recker, K. M., \& Strutt, J. (2011). Changes in children's perception-action tuning over short time scales: bicycling across traffic-filled intersections in a virtual 
environment. Journal of experimental child psychology, 108(2), 322-337. https://doi.org/10.10 16/j.jecp.2010.07.005

26. Stoffregen, T. A. (2018). Affordances as properties of the animal-environment system. In How Shall Affordances Be Refined? Four Perspectives (pp. 115-134): Routledge. https://doi.org/10.4324/9780203726655$\underline{2}$

27. Teo, A. R., \& Gaw, A. C. (2010). Hikikomori, a Japanese culture-bound syndrome of social withdrawal?: a proposal for DSM-5. The Journal of Nervous and Mental Disease, 198(6), 444-449. https://doi.org/10.1097/N MD.0b013e3181e086b1

28. Tom, A., \& Granie, M. A. (2011). Gender differences in pedestrian rule compliance and visual search at signalized and unsignalized crossroads. Accident Analysis \& Prevention, 43(5), 1794-1801. https://doi.org /10.1016/j.aap.2011.04.012

29. Warren, W. H. (2006). The dynamics of perception and action. Psychological Review, 113(2), 358-389. https://doi.org/10.1037/0033-295X.113.2.358

30. WHO. (2018). Global status report on road safety 2018 (9789241565684). Retrieved from https://www.who.int /publications/i/item/9789241565684

31. Zeng, N., Ayyub, M., Sun, H., Wen, X., Xiang, P., \& Gao, Z. (2017). Effects of physical activity on motor skills and cognitive development in early childhood: a systematic review. BioMed Research International, 2017, 2760716. https://doi.org/10.1155/2017/2760716 